\title{
Face Detection Based On Eye-Mouth Triangular Approach
}

\author{
Deni Kartika $^{1^{*}}$ and Suprijadi ${ }^{1}$ \\ ${ }^{1}$ Electronics and Instrumentation Laboratory, \\ Theoretical High Energy Physics and Instrumentation Research Group, \\ Faculty of Mathematics and Natural Sciences, Institut Teknologi Bandung, \\ Jl. Ganesha No. 10, Bandung 40132, Indonesia
}

( Received: August 29, 2019, Revised: February 28, 2020, Accepted: August 11, 2020 )

\begin{abstract}
Human face is a complex and dynamic structure. It is a challenge to be able to make a face recognition system like humans. At the beginning of its development, many facial recognition studies only focused on facial features. In 1991, Turk and Pentland developed a face recognition system based on Principal Component Analysis named eigenface. This system is very efficient because it only focuses on components that most affect facial image. However, this system has weaknesses, which cannot be used to determine the position of the face. In this final project, image processing methods will be carried out to detect faces in digital images. The method used is eye mouth triangular approach with the steps being taken are skin detection, eye detection, mouth detection, and facial confirmation. From the results of a hundred digital color images tested, there were 82 images that were successfully detected. The main system failure is caused by failure in skin detection. Further development is needed so that the system can work optimally.
\end{abstract}

Keywords: Face detection, eye-mouth triangular approach, image processing, digital color image

\section{INTRODUCTION}

Face recognition system is one application that is widely used in image analysis. A challenge in itself is to create an automated system that is equivalent to the way humans recognize faces. Although humans are very good at identifying known faces, they are not very skilled when dealing with a large number of unknown faces. Computers with almost unlimited memory and computing speed can overcome human limitations.

Developing a facial recognition system is a complex problem because the human face is a very complex and dynamic structure with characteristics that can change quickly and significantly every time. Initially, the method used for facial recognition systems generally focused on individual features of the face, such as eyes, nose, mouth, eyebrows, and headlines. From these results, individual features are then mapped on a two or three-dimensional model to produce detailed geometry. However, several studies have shown that facial recognition with this system is very fragile and sensitive to the factors of increasing age and changes in facial features. In addition, these methods also require large data and storage media.

The eigenface method is one of the face recognition methods developed by Turk and Pentland in 1991. The method represents the image efficiently using the Principal Component Analysis process. In this method, the face image will be projected in a feature space that features significant variations between the known face images. This significant feature has come to be referred to as "eigenface." Eigenface will only capture a number of points in the image that cause significant variations between faces in the database that make them different.

The eigenface method offers a more efficient and simpler system for recognizing faces. However, this method is only suitable for controlled images, in this case, it is only suitable for recognizing faces in an image of an appropriate size and loading a full face in one frame. The eigenface method is not suitable for finding where the face is.

\footnotetext{
* Corresponding author

E-mail address: kartika.deni@gmail.com
} 
This paper will present alternative techniques to overcome problems in the eigenface facial recognition system. By using a proper face detection system, the eigenface face recognition system will be an easy-to-use system for various image samples being tested.

\section{DIGITAL IMAGE PROCESSING}

\section{Digital Image Operations}

\section{Contour-Convex Hull}

Contour is a collection of connected points that represent curves in the image [1]. The representation can be different, depending on the conditions encountered.

The convex hull of a contour is the polygonal approach of the contour by ignoring the convex defects in the image. Examples of applications of the convex hull can be seen in Figure 1.

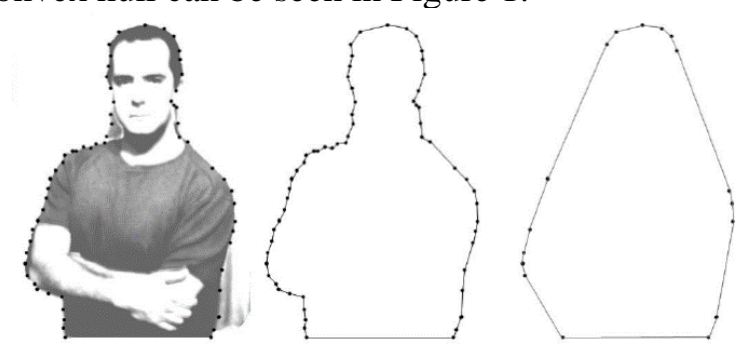

Figure 1. Convex hull application. Sequential images from left to right: original image, contour, convex hull from contour [1]

\section{Face Detection}

\section{Skin Detection}

The face detection process used in color processing. There are several conditions for the color to be classified as skin. The following conditions are used [2]:

$R>95$ and $G>40$ and $B>20$ and $R>G$

and $R>B$ and $|(R-G)|>15$

and $\max (R ; G ; B)-\min (R ; G ; B)>135$

and $C r>135$ and $C b>85$ and $Y>80$

and $\mathrm{Cr} \leq(1,5862 \times \mathrm{Cb})+20$

and $\mathrm{Cr} \geq(0,3448 \times \mathrm{Cb})+76,2069$

and $C r \geq(-4,5652 \times C b)+234,5652$

and $\mathrm{Cr} \leq(-1,15 \times \mathrm{Cb})+301,75$

and $C r \geq(-2,2857 \times C b)+432,85$.

\section{Eye Detection}

In the eye detection, Eye Map is used. The made eye map consists of two separate parts, one for the chrominance component and the other for the luminance component [3]. Eye map of chroma based on observations with high $\mathrm{Cb}$ and low $\mathrm{Cr}$ values found around the eyes. Formulated as

$$
\text { EyeMapC }=\frac{1}{3}\left\{\left(C b^{2}\right)+(\widetilde{C r})^{2}+\left(\frac{C b}{C r}\right)\right\}
$$

with $\left(C b^{2}\right),(\widetilde{C r})^{2},\left(\frac{C b}{C r}\right)$, everything has been normalized in the range $[0 ; 255]$ and $\widetilde{C r}$ is a negative component of $\mathrm{Cr}$ (that is, $255-\mathrm{Cr}$ )

In the luminance component, the eye is generally composed of dark and bright pixels. Gray morphological operators, namely dilation and erosion can be designed to emphasize lighter and darker pixels in the luma components around the eye area. This operator has been used to create feature vectors for faces at various scales for frontal face authentication. The equation for creating a Luminance Eye Map is [4]

$$
\text { EyeMapL }=\frac{Y(x, y) \oplus g_{\sigma}(x, y)}{Y(x, y) \ominus g_{\sigma}(x, y)+1}
$$

with

$\begin{array}{lllllll}0,7498 & 1,1247 & 1,4996 & 1,8745 & 1,4996 & 1,1247 & 0,7498\end{array}$

$\begin{array}{lllllll}1,1247 & 1,4996 & 1,8745 & 2,2494 & 1,8745 & 1,4996 & 1,1247\end{array}$

$\begin{array}{lllllll}1,4996 & 1,8745 & 2,2494 & 2,6243 & 2,2494 & 1,8745 & 1,4996\end{array}$

$\begin{array}{lllllll}g_{\sigma}=1,8745 & 2,2494 & 2,6243 & 2,9992 & 2,6243 & 2,2494 & 1,8745\end{array}$

$\begin{array}{llllllll}1,4996 & 1,8745 & 2,2494 & 2,6243 & 2,2494 & 1,8745 & 1,4996\end{array}$

$\begin{array}{llllllll}1,1247 & 1,4996 & 1,8745 & 2,2494 & 1,8745 & 1,4996 & 1,1247\end{array}$

$\begin{array}{lllllll}0,7498 & 1,1247 & 1,4996 & 1,8745 & 1,4996 & 1,1247 & 0,7498\end{array}$

$\bigoplus$ is dilation and $\ominus$ is erosion.

After obtaining the Chrominance Eye Map and Luminance Eye Map, then collected with the operation AND (multiplication).

$$
\text { EyeMap }=(\text { EyeMapC }) \text { AND }(\text { EyeMapL }
$$

\section{Mouth Detection}

Color in the mouth region consists of a stronger red component and a weaker blue component than other skin areas [5]. Thus, the $\mathrm{Cr}$ chrominance component is greater than the $\mathrm{Cb}$ chrominance component in the mouth area. The mouth region also has a relatively low response to $\left(\frac{C r}{C b}\right)$ and a high response to $C r^{2}$. Mouth Map is designed as follows

$$
\begin{aligned}
\text { MouthMap } & =C r^{2} \cdot\left(C r^{2}-\eta \cdot\left(\frac{C r}{C b}\right)\right. \\
\eta & =0,95 \cdot \frac{\frac{1}{n} \Sigma C r^{2}}{\frac{1}{n} \Sigma\left(\frac{C b}{C r}\right)},
\end{aligned}
$$

\section{Face Confirmation Using Eye-Mouth Triangle Geometry}

After obtaining an Eye Map along with a Mouth Map of all possible candidates, take three points $i, j$, and $k$ which can form a triangle [3]. Define $i$ is the center of the left eye block, $j$ is the center of the right eye block, and $k$ is the center of 
the mouth block. There are four rules that are suitable for isosceles triangles, namely:

$$
\begin{aligned}
& |D(i, j)-D(j, k)|<0,25 \times \max (D(i, j), D(j, k)) \\
& |D(i, j)-D(i, k)|<0,25 \times \max (D(i, j), D(i, k))
\end{aligned}
$$

for coordinates $x, i<j$ and $k<j$, while for coordinates $y, i<j<k$. With, $D(a, b)$ shows the Euclidean distance between the center points of block $a$ and block $b$.

\section{SYSTEM DESIGN AND IMPLEMENTATION}

Theory section should extend, not repeat, the background to the article already dealt with in the Introduction and lay the foundation for further work. In contrast, a Calculation section represents a practical development from a theoretical basis.

\section{THEORY/CALCULATION (if any)}

\section{System Workflow}

The following is a flow chart of the system worked on in this study.

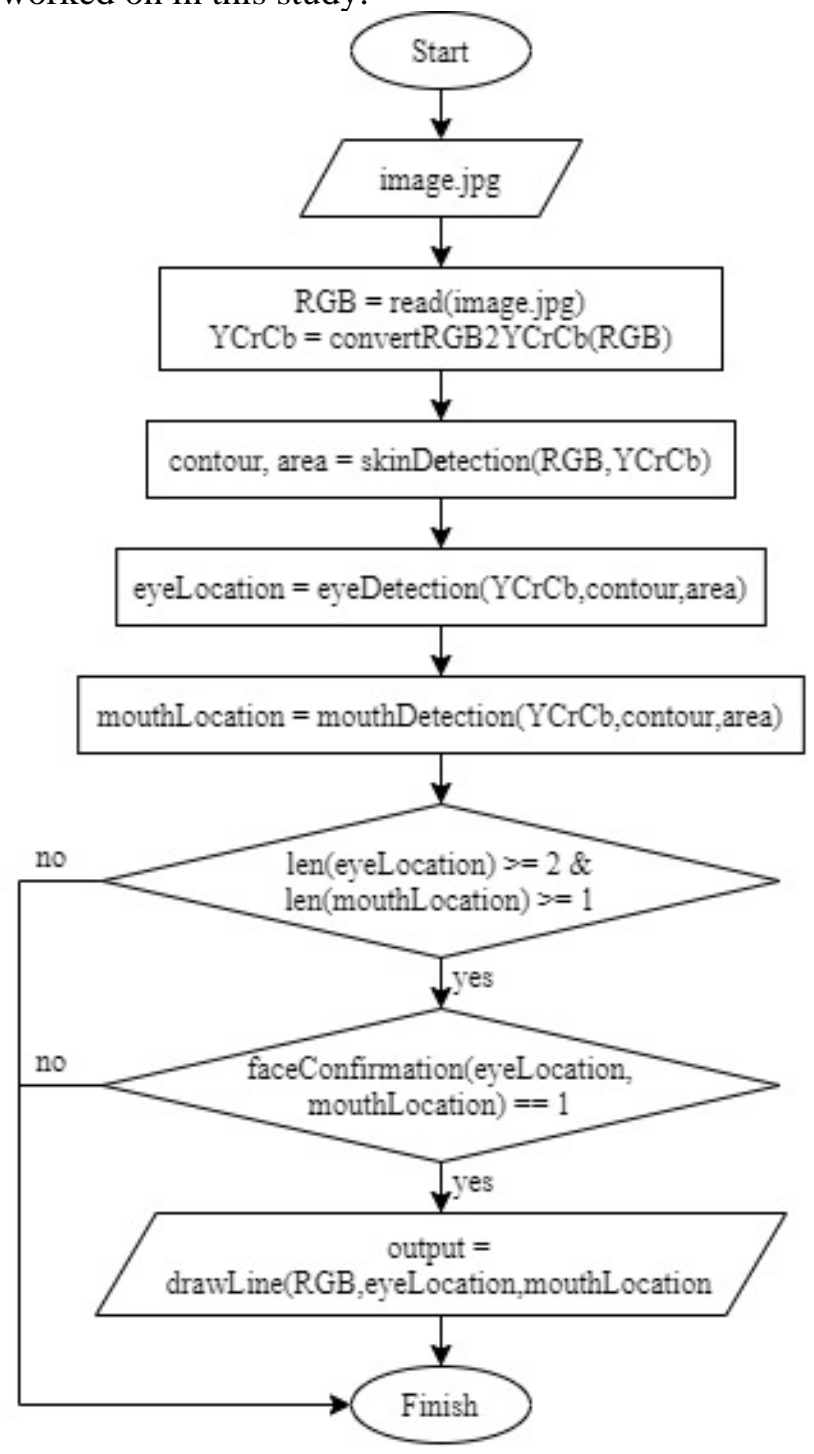

Figure 2. System flow chart.
System input is an image that contains face data and the system output is a decision about the facial identity in that image. The overall system implementation uses Python 3.7 software with OpenCV 3.4.4 library as the main platform. In the next section, we will explain the process of detecting and recognizing faces that are run by the system in more detail.

\section{Face Detection}

The face detection system functions to detect faces using the eye-mouth triangle approach. In the system, there are stages of eye detection, mouth detection, and face confirmation which are discussed in the next section.

An example of the input image is shown in Figure 3. The input image will be processed in the system. The output of the system is exemplified in Figure 4.

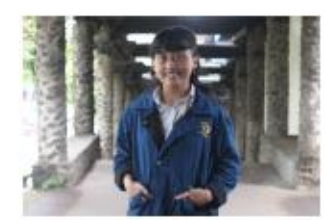

Figure 3. System input in the form of a color digital image.

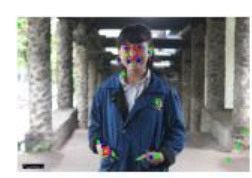

(a)

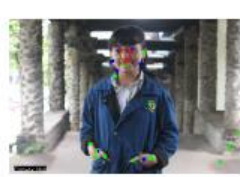

(b)

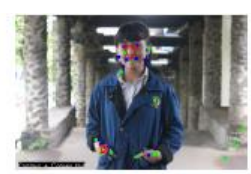

(c)
Figure 4. System output (a) skin area contour mask, (b) convex hull mask skin area, (c) contour mask + convex hull mask.

\section{Skin Detection}

Skin detection is an operation to select the portion of the input image that is human skin and not human skin. The stage of skin detection can be seen in Figure 5.

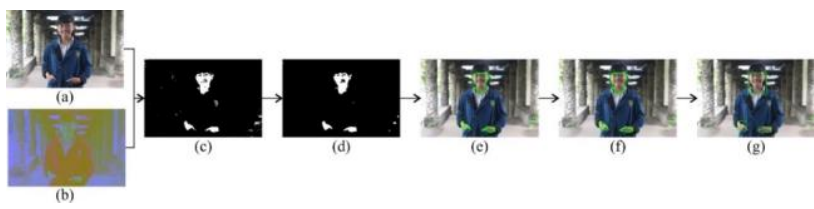

Figure 5. Skin detection stage, (a) RGB input image, (b) $\mathrm{YCrCb}$ input image, (c) thresholding result image, (d) processed thresholding image, (e) face contour, (f) convex face hull, (g) face candidates.

\section{Eye Detection}

Eye detection is an operation to find the location of the eye on a candidate's face that has been obtained. The stage of eye detection can be seen in Figure 7 and Figure 8. 


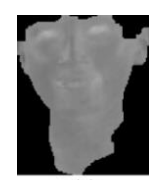

(a)

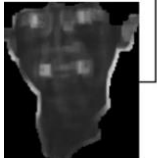

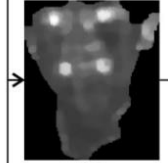

(c)

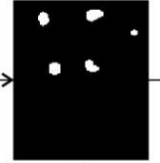

(d)

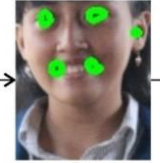

(e)

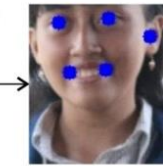

(f) (b)

Figure 6. Step of eye detection using contour mask, (a) chrominance eye map, (b) luminance eye map, (c) eye map, (d) eye area thresholding, (e) eye area contour, (f) candidate eye location.

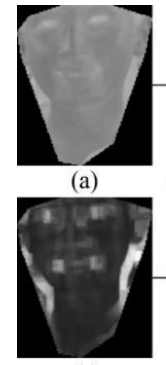

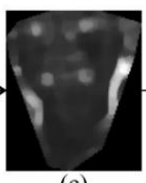

(c)

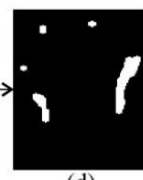

(d)

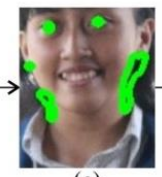

(e)

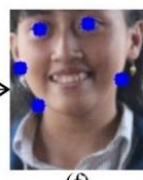

(f) (b)

Figure 7. Step of mask eye detection using convex hull mask, (a) chrominance eye map, (b) luminance eye map, (c) eye map,

(d) eye area thresholding, (e) contour eye area, (f) candidate location.

\section{Mouth Detection}

Mouth detection is an operation to find the location of the mouth on a candidate's face that has been obtained. The stage of mouth detection can be seen in Figure 9 and Figure 10.

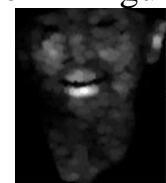

(a)

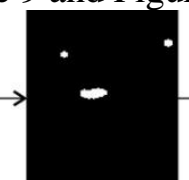

(b)

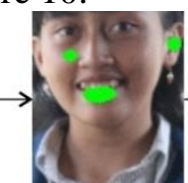

(c)

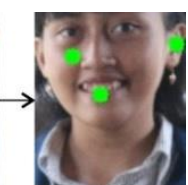

(d)
Figure 8. Step of mouth detection using contour mask, (a) mouth map, (b) thresholding of mouth area, (c) contour mouth area, (d) location of mouth candidate.

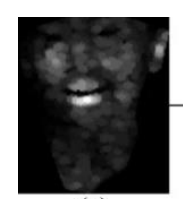

(a)

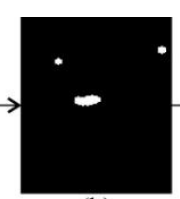

(b)

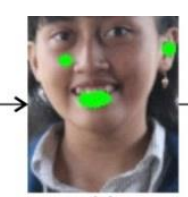

(c)

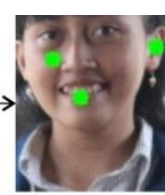

(d)
Figure 9. Step of mask mouth detection using convex hull mask, (a) mouth map, (b) thresholding of mouth area, (c) contour of mouth area, (d) location of candidate's mouth.

\section{Face Confirmation Using Eye-Mouth Triangle Geometry}

Confirmation of face candidates using predetermined eye geometry and triangle orientation between eyes. The step of facial confirmation using eye-mouth geometry can be seen in Figure 10 and Figure 11.

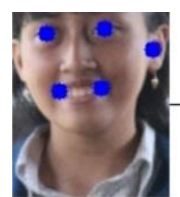

(a)

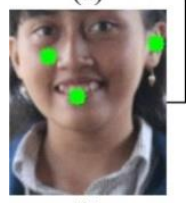

(b)

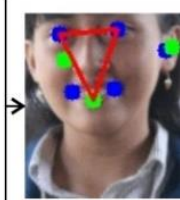

(c)
Figure 10. Step of face confirmation stage using contour mask eye-mouth geometry, (a) location of candidate's eyes, (b) location of candidate's mouth, (c) face confirmation.

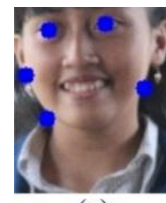

(a)

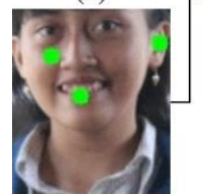

(b)

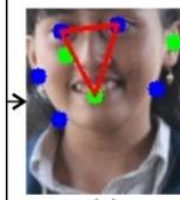

(c)
Figure 11. Step of face confirmation using convex hull mask eye-mouth geometry, (a) location of the candidate's eyes, (b) location of the candidate's mouth, (c) facial confirmation.

\section{TESTING AND ANALYSIS}

\section{Test Results}

System testing is carried out on one hundred test images measuring $5184 \times 3456$ that have been provided. System testing is done to see the performance of the face detection system that has been made. In testing, masking variations are performed on the image, using contour and convex hull and the combination of the two. The following system test results in table 1 , and table 2 .

Table 1. Performance of face detection system with contour mask

\begin{tabular}{lllll}
\hline Step & Succeed & Failed & Sample & DR $(\%)$ \\
\hline Skin Detection & 43 & 57 & 100 & 43,0 \\
\hline Eye Detection & 41 & 2 & 43 & 95,3 \\
$\begin{array}{l}\text { Mouth } \\
\text { Detection }\end{array}$ & 38 & 5 & 43 & 88,7 \\
\hline $\begin{array}{l}\text { Triangular } \\
\text { Confirmation }\end{array}$ & 35 & 2 & 37 & 94,6 \\
\hline \hline Face Detection & 35 & 65 & 100 & 35,0 \\
\hline
\end{tabular}

Face detection using a contour mask uses the contour application to maintain skin tone in the contour area and make other zero values in the image. In the table section, the number of samples at the skin detection stage is the number of test images, at the eye detection and mouth detection stage is the 
number of successful skin detection, while at the face confirmation stage is the number of eye and mouth detection successfully.

Table 2. Performance of face detection system with convex hull mask

\begin{tabular}{lllll}
\hline Step & Succeed & Failed & Sample & DR $(\%)$ \\
\hline Skin Detection & 99 & 1 & 100 & 99,0 \\
\hline $\begin{array}{l}\text { Eye Detection } \\
\begin{array}{l}\text { Mouth } \\
\text { Detection }\end{array}\end{array}$ & 90 & 29 & 99 & 70,7 \\
\hline $\begin{array}{l}\text { Triangular } \\
\text { Confirmation }\end{array}$ & 63 & 6 & 66 & 95,4 \\
\hline \hline Face Detection & 63 & 37 & 100 & 63,0 \\
\hline
\end{tabular}

Face detection using the convex hull mask of skin contour to maintain skin tone in the convex hull region and make the other zero value in the image. In the table section, the number of samples at the skin detection stage is the number of test images, at the eye detection and mouth detection stage is the number of successful skin detection, while at the face confirmation stage is the number of eye and mouth detection successfully.

Theory section should extend, not repeat, the background to the article already dealt with in the Introduction and lay the foundation for further work. In contrast, a Calculation section represents a practical development from a theoretical basis.

\section{Skin Detection}

Skin detection is the earliest stage carried out in the face detection process. The definition of skin color has followed the values determined by Kolkur et al in Human Skin Detection Using RGB, HSV and YCbCr Color Models, 2017, but there are still many errors. From the use of contour, there were 57 errors from one hundred images tested. Errors that occur because there is a shadow or light on the skin that causes the skin is not detected optimally which causes the eye area missed from detection. The use of convex hull is expected to reduce these defects, by eliminating the hollows formed due to undetected eye area, to be more clearly seen in figure 14 . However, there is a failure of the convex hull in detecting the skin, because the contour is converted to the convex hull in very bad condition, as can be seen in figure 15.

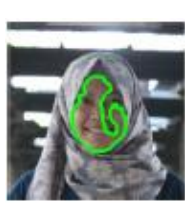

(a)

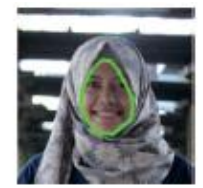

(b)
Figure 12. (a) Failed contour skin detection, (b) successful detection of convex hull skin.

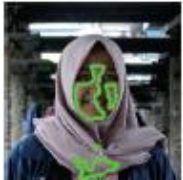

(a)

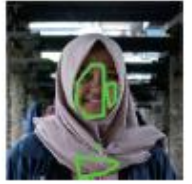

(b)
Figure 13. (a) Failed contour skin detection, (b) failed convex hull skin detection.

\section{Eye Detection}

Eye detection functions to determine the location of the candidate's eyes on the image. In the system created, a maximum of five candidate locations in the image will be searched. Regardless of the failure in skin detection, in the contour mask, the success of the system in detecting the eyes is quite high with a success rate of $95.3 \%$. The success rate drops to $70.7 \%$ when convex hull is used, this is because the use of convex hull will eliminate the hollow in the area near the ear, neck, and so on which results in a candidate eye being more dominant than the actual eye (the definition of an eye has a highintensity transition and low which is also found in the transference of the skin or the edge of the skin against a light background)

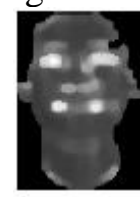

(a)

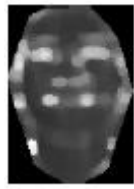

(e)

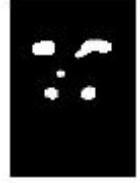

(b)

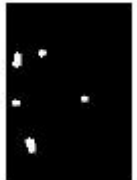

(f)

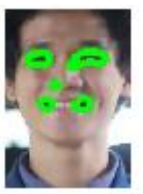

(c)

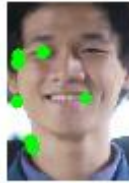

(g)

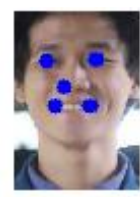

(d)

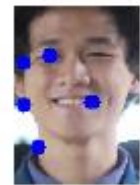

(h)
Figure 14. Successful detection of contour eyes (a) eye map, (b) threshold, (c) contour, (d) candidate location; failed detection of convex hull eyes (e) eye map, (f) threshold, (g) contour, (h) candidate location

\section{Mouth Detection}

The mouth detection function is to determine the location of the mouth candidate in the image. In the system created, a maximum of three candidate locations of the mouth will be sought in the image. For both methods of masking, contour and convex hull there are no significant problems. Mistakes that occur quite a bit and are somewhat unique, namely when the skin detection system detects objects alongside the skin with a color that is detected as skin color and there is a dominant red chrome color (mouth definition) in it. An example can be seen in figure 15 . 


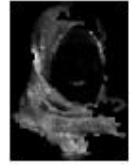

(a)

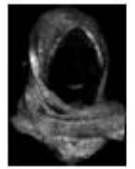

(e)

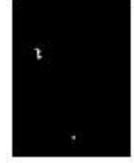

(b)

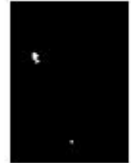

(f)

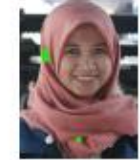

(c)

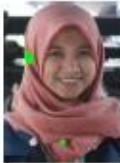

(g)

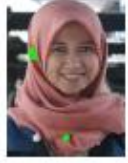

(d)

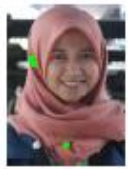

(h)
Figure 15. Failed mouth detection of contour (a) eye map, (b) threshold, (c) contour, (d) candidate location; failed detection of mouth convex hull, (e) eye map, (f) threshold, (g) contour, (h) candidate location.

\section{Face Confirmation Using Eye-Mouth Triangle Geometry}

Face confirmation functions to select candidates from the eyes and mouth that have been obtained from the previous stage which can be formed by a face with predetermined conditions. From the test results, there were no significant problems. The success rate for the two masking methods used is already high, above $90 \%$. However, the conditions used for facial confirmation are still very rough and can be developed further.

\section{Face Detection}

From the face detection that has been done, following the final tabulation of the face detection results using masking variations. Total faces detected using eyes and mouth were detected in the contour masking method and/or convex hull which was then combined in the eye-mouth triangle confirmation. The tabulation of results can be seen in Table 4 .

Table 4. Differences in performance of facial detection systems by masking contour, convex hull, and total detected faces.

\begin{tabular}{llll}
\hline Masking Method & Succeed & Failed & DR(\%) \\
\hline Contour & 35 & 65 & 35,0 \\
Convex hull & 63 & 37 & 63,0 \\
\hline \hline Total faces detected & 82 & 18 & 82,0 \\
\hline
\end{tabular}

In the form of a Venn diagram, the distribution of the success of face detection of contour mask and convex hull mask in a combination of contour mask and convex hull mask can be seen in Figure 16.

In Figure 16 it can be seen that 35 faces were detected in the contour masking method and 63 faces were detected in the convex hull masking method. There are 18 slices of images that have successfully detected a face in both masking methods. There are 2 images that have successfully detected a face through the merging of the eyes and mouth detected in the masking contour and convex hull method.

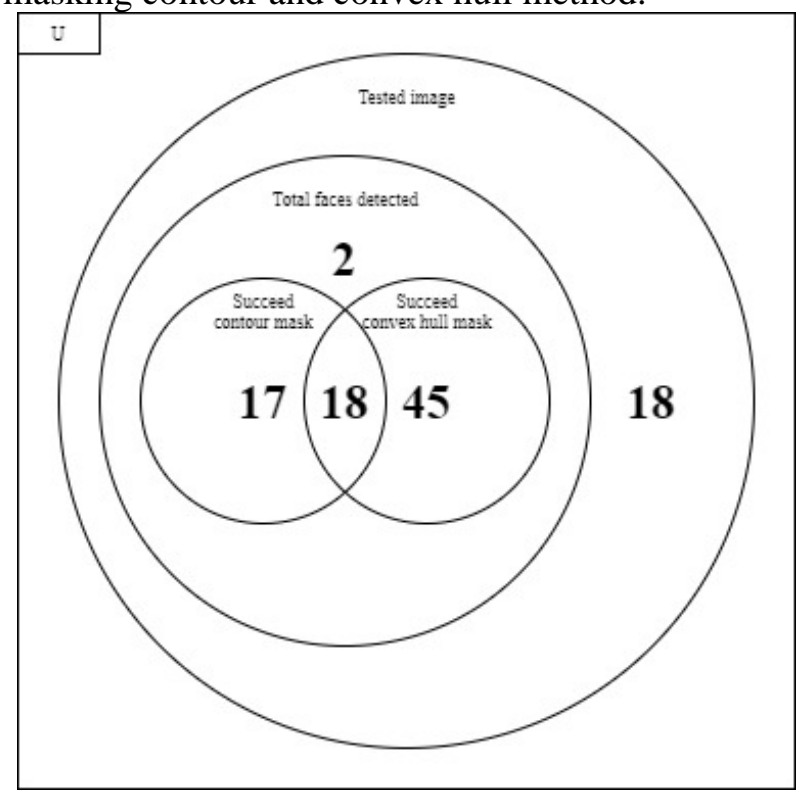

Figure 16. A diagram of a summary of system performance.

\section{CONCLUSION}

Based on research that has been done, the conclusion obtained is that the face detection system based on the eye-mouth triangle approach that has been made can work well with a success rate of $82 \%$ for a test image of one hundred pieces.

\section{REFERENCES}

[1] Kaehler, A., \& Bradski, G., Learnig opencv 3: Computer vision in c++ with the opencv library. O'Reilly, California, 2017.

[2] Kolkur, S., Kalbande, D., Shimpi, P., Bapat, C., \& Jatakia, J., Human Skin Detection Using RGB, HSV and YCbCr Color Models, ICCASP/ICMMD Advances in Intelligent Systems Research, 137, 324-332, 2016.

[3] Rahman, M. H., Jhumur, F., Yusuf, M. S. U., Das, T., \& Ahmad, M., An Efficient Face Detection in Color Images Using Eye Mouth Triangular Approach, IEEE/OSA/IAPR International Conference on Informatics, Electronics and Vision, 530-535, 2012.

[4] Sheu, J.-S., Hsieh, T.-S., \& Shou, H.-N., Automatic Generation of Facial Expression Using Triangular Geometric Deformation, Journal of Applied Research and Technology, 12, 1115-1130, 2014.

[5] Hsu, R.-L., Abdel-Mottaleb, M., \& Jain, A. K., Face Detection in Color Images, IEEE Transactions on Pattern Analysis and Machine Intelligence, 24 (5), 696-706, 2002. 